\title{
Infliximab-Induced Linear Iga Bullous Disease in a Patient with Ulcerative Colitis
}

\author{
Nadia K Sundlass, Nicholas T Woltjen, Chinmoy Bhate, Jonhan Ho and Joseph C. English II* \\ Department of Dermatology, University of Pittsburgh, Pittsburgh, USA
}

\begin{abstract}
Linear Iga Bullous Disease (LABD) is an autoimmune blistering disease that can occur spontaneously or secondary to medications. Recently LABD has been reported in association with ulcerative colitis in which treatment with infliximab resolved both diseases. We describe a patient with ulcerative colitis who developed LABD disease after exposure to infliximab therapy for ulcerative colitis.
\end{abstract}

Keywords: Linear Iga Bullous disease; Autoimmune, Infliximab therapy; Ulcerative colitis

\section{Case Report}

A 28 year old white male with ulcerative colitis on 40 mg prednisone was started on infliximab as a Steroid-sparing agent. Approximately 5 $1 / 2$ weeks after initiating infliximab, he developed itchy pustules on his trunk. The patient was started on doxycycline $100 \mathrm{mg}$ twice daily for presumed steroid- induced acne. The patient received the 3rd infusion of infliximab 4 days later and immediately developed rapidly-spreading, pruritic bullae on the head, neck, trunk, back, abdomen, genitalia, and all extremities. He presented to the dermatology clinic 1 week later with numerous polycyclic, tense persistent bullae clustered in a pattern resembling a "crown of jewels," with central crusting [1,2] (Figures 1-3).

Skin biopsies of lesional and perilesional skin were submitted for

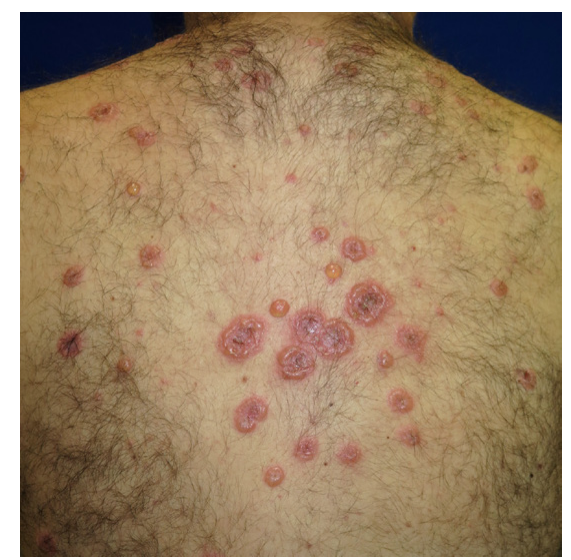

Figure 1: Linear Ig A bullous disease due to Infliximab. Polycyclic tense bullae.

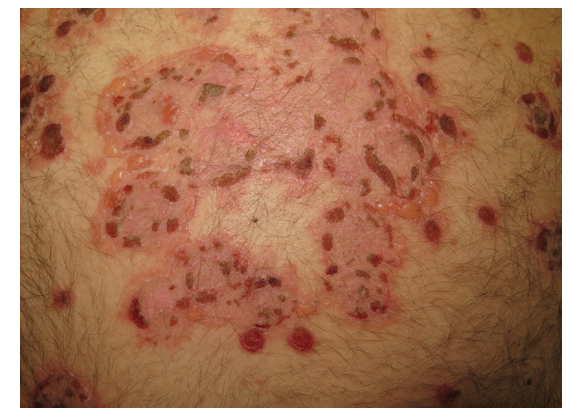

Figure 2: Linear IgA bullous disease due to Infliximab. Confluent lesions.

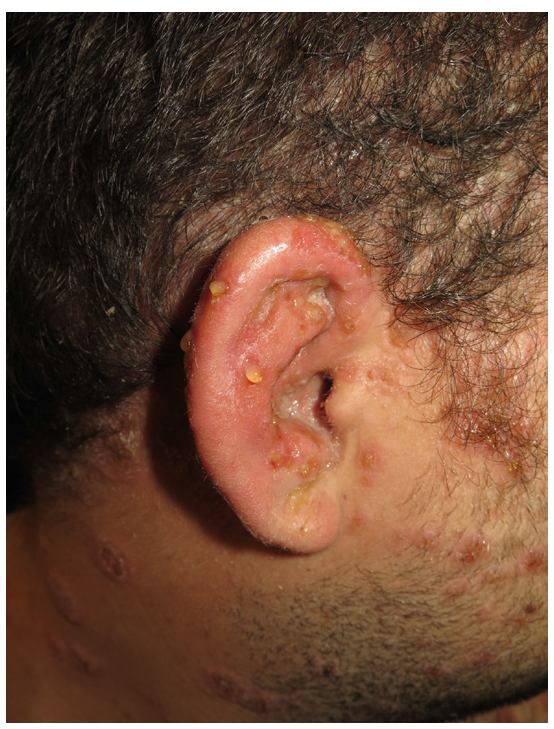

Figure 3: Linear IgA bullous disease due to Infliximab. R. ear involvement.

$\mathrm{H} \& \mathrm{E}$ and direct immunofluorescence (DIF) studies. The histologic sections demonstrated a subepidermal blister with neutrophils as well as linear deposits of IgA and weak deposits of IgG along the Dermoepidermal Junction (DEJ) (Figures 4 and 5). A diagnosis of infliximab induced LABD was made based on the clinical findings, timeline of drug exposures, and histopathological changes. Infliximab infusions and oral doxycycline were stopped. The patient required an increase to $80 \mathrm{mg}$ oral prednisone daily to control his LABD and he was started on vedolizumab, a monoclonal antibody targeting integrin a $4 \beta 7$ that was recently approved for ulcerative colitis, after a 8 week washout period from the last infliximab infusion. Due to a reported sulfa allergy in this patient, he underwent rapid desensitization and is

*Corresponding author: Joseph C English, Department of Dermatology, University of Pittsburgh, Pittsburgh, USA, Tel: +1 412-624-4141; E-mail: englishjc@upmc.edu

Received August 22, 2015; Accepted September 11, 2015; Published September 18,2015

Citation: Sundlass NK, Woltjen NT, Bhate C, Ho J, English JC (2015) InfliximabInduced Linear Iga Bullous Disease in a Patient with Ulcerative Colitis. J Clin Case Rep 5: 597. doi:10.4172/2165-7920.1000597

Copyright: (c) 2015 Sundlass NK, et al. This is an open-access article distributed under the terms of the Creative Commons Attribution License, which permits unrestricted use, distribution, and reproduction in any medium, provided the original author and source are credited. 


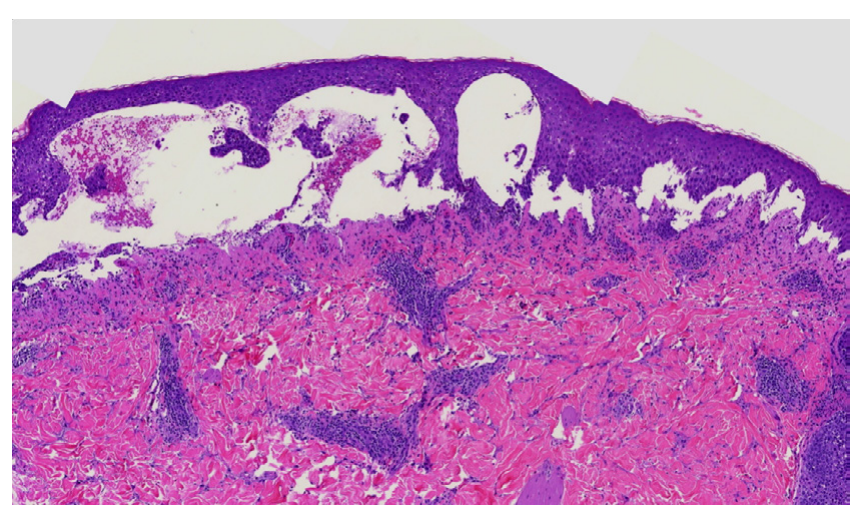

Figure 4: Histopathology demonstrating subepidermal blister with neutrophilic-rich infiltrate (H\&E x5).

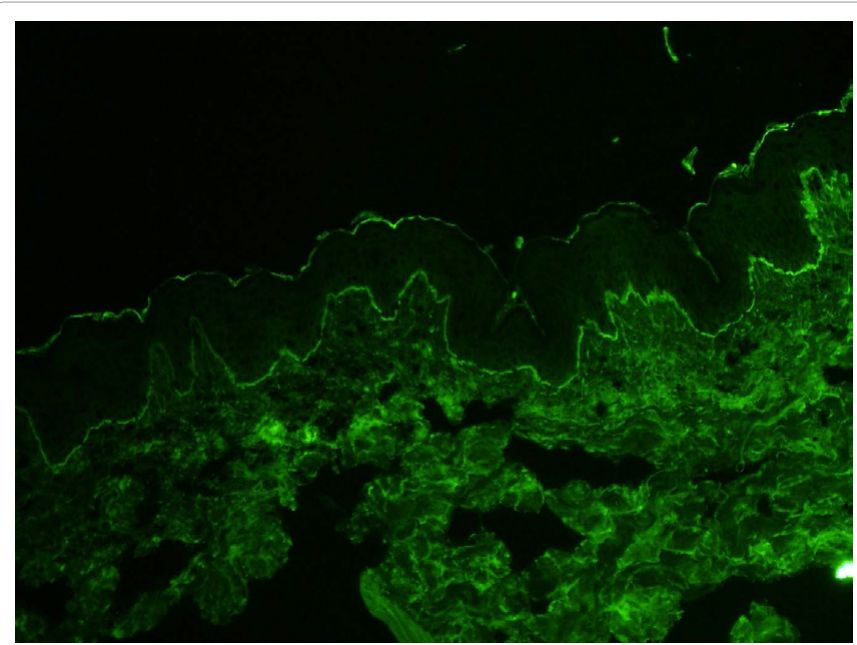

Figure 5: Direct immunofluorescence demonstrating linear deposits of IgA along the basement membrane zone (DIF x10).

currently on $175 \mathrm{mg}$ dapsone daily to treat the associated LABD. The patient's ulcerative colitis and linear IgA disease have been successfully controlled with vedolizumab and oral dapsone, respectively. He remains disease free after having tapered off systemic corticosteroids.

\section{Discussion}

LABD is an autoimmune bullous disorder of the skin, with antibodies targeting the lamina lucida, the sublamina densa, or both [3]. Its incidence is reported to be less than 1 case per million and it occurs in both children and adults. LABD may occur spontaneously, secondary to a drug (most commonly implicated is vancomycin) or, as has been described recently, in the setting of ulcerative colitis $[1,2]$. The typical clinical presentation is a polycyclic grouping of bullae with central crusting, often referred to as a "crown of jewels" pattern. DIF demonstrates linear deposits of IgA along the DEJ [1]. Management typically involves termination of any potentially implicated medications and initiation of systemic corticosteroids and oral dapsone [4]. Druginduced LABD is usually thought to be more severe than spontaneous LABD, and usually starts within 4 weeks of medication initiation and resolves quickly after drug withdrawal [1], however, this observation is based upon medications with therapeutic levels that are achieved within days, rather than weeks, such as infliximab.
Anti-Tumor Necrosis Factor-Alpha (TNF) agents are efficacious in a broad spectrum of autoimmune disorders such as ulcerative colitis, rheumatoid arthritis, and psoriasis [2]. TNF antagonists may also paradoxically induce autoimmune diseases after months to years of therapy, such as lupus, which persist for months after withdrawal [5]. Infliximab was recently reported to induce pemphigus foliaceus; adalimumab was implicated in a case of bullous pemphigoid [6]. This is hypothesized to be secondary to suppression of Th1 cytokines by TNF antagonists, with a resulting shift towards Th2 cytokine production and production of autoantibodies [5]. Our case suggests that infliximab may also induce LABD. We considered that our patient developed LABD secondary to his underlying ulcerative colitis rather than medication usage; however, this typically occurs in patients with uncontrollable disease [7]. Our patient's gastrointestinal symptoms were well-controlled throughout the development and progression of the LABD. Secondly, the patient began to develop blisters after the 2 nd infusion, with rapid progression following the 3rd infusion, arguing for a drug association. While drug-induced LABD has historically occurred within 4 weeks of drug initiation, we argue that anti-TNF agents can cause autoimmune diseases weeks to years after drug initiation and can last up to 6 months before improving. Given the extensive surface area involvement of our patient and extended half-life of infliximab, we treated aggressively with steroids and oral dapsone to minimize complications due to both LABD and long-term steroid use. To our knowledge, this is the second report of infliximab-induced LABD [8]. Anti-TNF agents are increasingly being used for a wide variety of autoimmune conditions, and awareness of paradoxical side effects is of clinical relevance.

\section{References}

1. Chanal J, Ingen-Housz-Oro S, Ortonne N, Duong T-A, Thomas M, et al. (2013) Linear IgA bullous dermatosis: comparison between the drug-induced and spontaneous forms. Brit J Dermatol 169: 1041-1048.

2. Yamada S, Makino T, Jinnin M, Sakai K, Fukushima S, et al. (2013) Association of Linear IgA Bullous Disease with Ulcerative Colitis: A Case of Successful Treatment with Infliximab. Dermatology 227: 295-298.

3. Zone J, Taylor T, Meyer L, Petersen M (1998) The $97 \mathrm{kDa}$ linear IgA bullous disease antigen is identical to a portion of the extracellular domain of the 180 kDa bullous pemphigoid antigen BPAg2. J Invest Dermatol 10: 207-210.

4. Verma R, Vasudevan B, Sagar A, Pragasam V, Deb P, et al. (2013) A case of linear immunoglobulin $A$ disease with dapsone hypersensitivity and its management strategies. Indian J Dermatol. 79: 833-835.

5. Almoallim H, Al-Ghamdi Y, Almaghrabi H, O Alyasi (2012) Anti-Tumor Necrosis Factor-alpha Induced Systemic Lupus Erythematosus. Open Rheumatol J 6: 315-319.

6. Boussemart L, Jacobelli S, Batteux F, Goulvestre C, Grange P, et al. (2010) Autoimmune bullous skin diseases occurring under anti-tumor necrosis factor therapy: two case reports. Dermatology 221(3): 201-205.

7. Watchorn R, Ma 2, Gulmann C, Keogan M, O'Kane M (2014) Linear IgA disease associated with Ulcerative Colitis: The role of surgery. Clin Exp Dermatol 39: 327-329.

8. Hoffman J, Hadaschik E, Enk A, Stremmel W, Gauss A (2015) Linear IgA Bullous Dermatosis secondary to infliximab therapy in a patient with Ulcerative Colitis. Dermatology 231: 112-115. 